\title{
Investigation of Thermal Losses in a Soft Magnetic Composite Using Multiphysics Modelling and Coupled Material Properties in an Induction Heating Cell
}

\author{
Leif Siesing, Kenneth Frogner, Tord Cedell, Mats Andersson \\ Division of Production and Materials Engineering, Lund University, Lund, Sweden \\ Email: leif.siesing@iprod.lth.se
}

How to cite this paper: Siesing, L., Frogner, K., Cedell, T. and Andersson, M. (2016) Investigation of Thermal Losses in a Soft Magnetic Composite Using Multiphysics Modelling and Coupled Material Properties in an Induction Heating Cell. Journal of Electromagnetic Analysis and Applications, 8, 182196.

http://dx.doi.org/10.4236/jemaa.2016.89018

Received: July 16, 2016

Accepted: September 23, 2016

Published: September 26, 2016

Copyright $\odot 2016$ by authors and Scientific Research Publishing Inc. This work is licensed under the Creative Commons Attribution International License (CC BY 4.0).

http://creativecommons.org/licenses/by/4.0/

\begin{abstract}
The complex interaction between material properties in an induction heating circuit was studied by multi physics simulation and by experimental verification in a fullscale laboratory heater. The work aims to illustrate the complexity of the system of interacting materials, but also to propose a method to verify properties of soft magnetic composite materials in an integrated system and to identify which properties are the most critical under different circumstances and load cases. Heat losses at different loads were primarily studied, from DC currents to AC currents at 15, 20 and $25 \mathrm{kHz}$, respectively. A FE model for magnetic simulation was correlated with a corresponding model for heat simulation. The numerical model, as well as the established input material data, could be verified through the experimental measurements. In this particular study, the current loss in the litz wire was the dominant heat source, thus making the thermal conductivity of the SMC the most important property in this material.
\end{abstract}

\section{Keywords}

Soft Magnetic Moldable Composite, Multiphysics, Litz, Induction Heating,

Thermal Losses, Coupled Material Properties

\section{Introduction}

Soft magnetic materials play a very important role in most electromagnetic devices, mainly to control the magnetic flux. The demands on the soft magnetic material may vary depending on application, but low internal losses are always a requirement. 
Typically, these materials range from laminated structures to different kinds of powderbased materials, often called soft magnetic composites (SMCs). This work involves one type of SMC material developed by the authors, but the results of the system analysis should be regarded as generic.

Ideally, a well-designed electromagnetic system should have no or very low internal energy losses, but this is the seldom case in practical applications [1]. Thermal losses in both coil and SMCs must be handled by different means. The thermal conductivity of the SMC material is important in this respect, but the thermal coupling between coil and SMC structure is also critical in order to facilitate cooling of the coil through the SMC material. There are studies that show that in many cases most of the heat is generated in the coil [2].

Each important material property at different load conditions, for example current levels and frequencies, can be determined by established methods for the coil material, SMC material and isolation materials [3]-[5]. However, there are always uncertainties in each characterization method [6] [7], and the required accuracy needed to perform accurate system simulations is always an issue [8]. It is also obvious that different applications, from electric drives to induction heating devices, put different demands on the combinations of material properties, and thus the choice of optimal material combinations. This study focuses on one such system, an induction heating device, which is an example of a complex system where the SMC material must be chosen and designed based on the integrated functionality. The importance of a multiphysics approach will be demonstrated, where the coupled effects of different material properties will be demonstrated. Numerical simulation through Finite Element Analysis (FEA) modeling is a powerful tool to analyze interacting and connected physical phenomena in an induction heater [9]-[11]. However, in order to verify the output from the simulation and to identify where poor material data input will be critical, a full-scale laboratory heater was built.

The laboratory induction heater was built with state-of-the-art technology, using litz wire in order to minimize losses in the current conductor, and using a specially developed powder-based soft magnetic material in order to minimize iron losses but also to facilitate optimal thermal coupling between coil and SMC body. The typical current frequency is set to $15-25 \mathrm{kHz}$, mainly due to the power system, which justifies the choice of material for this particular setup. An induction heater with these materials is normally designed for industry applications, such as heating steel belts, rods, rollers and sheets. The SMC material chosen has some special features, perhaps the most important one is the capability to be molded at room temperature into any given shape and size, thus given the name Soft Magnetic Moldable Composite $\left(\mathrm{SM}^{2} \mathrm{C}\right)$. This material has been developed by the authors at Lund University [12].

The $\mathrm{SM}^{2} \mathrm{C}$ material consists of two components: binder and soft magnetic powder. The binder is an epoxy and the powder is based on a $6.5 \%$ silicon-alloyed iron. The powder is gas atomized and has a particle size range of $10-400 \mu \mathrm{m}$. The material is molded and not pressed like conventional SMCs, which means that the particles are not deformed during manufacturing. There are a few advantages with this material. Firstly, 
the material will become isotropic, in other words the magnetic field can be directed in any direction in the material. Secondly, the material can be molded to just about any size and geometry (many other materials are limited in size, for instance). Thirdly, it is possible to get a good contact between the $\mathrm{SM}^{2} \mathrm{C}$ and other materials, resulting in good thermal contact. A good contact between electrical conductor and $\mathrm{SM}^{2} \mathrm{C}$ can result in leakage currents through the $\mathrm{SM}^{2} \mathrm{C}$ material. This is something that must be avoided and can be managed by applying an insulator material between the coil and $\mathrm{SM}^{2} \mathrm{C}$ material. This, however, decreases the thermal contact, which is undesirable. The most important properties of the $\mathrm{SM}^{2} \mathrm{C}$ material is the permeability, iron loss and thermal conductivity. All of these properties have been measured on different geometries, presented in [12]. Even if the model and the laboratory heater are based on this $\mathrm{SM}^{2} \mathrm{C}$ material, the presented methodology and the scientific approach will be shown to be generic, and any material combination can be studied and analyzed. Therefore, hereafter the soft magnetic material will simply be called SMC.

As mentioned earlier, the complex interaction between litz material, isolation materials and SMC materials sets the limits for the configurations of each of them. In order to obtain a complete model, the properties of the coil material, the internal current losses and thermal conductivity of the litz wire must be known. Models to calculate losses exist, and the thermal conductivity can be calculated by methods presented in [13].

The presented work uses a multiphysics approach in order to handle the complexity of a system with many interacting materials. A novel methodology to verify properties of soft magnetic materials in an integrated system by use of coupled simulation and experimental verification is presented. An additional objective in relation to this is to be able to identify which material properties are most critical under different circumstances and load cases.

\section{Method}

It is necessary to choose a geometry that will be suitable for both simulation and physical tests. By choosing a geometry with axisymmetric properties, a 2D model can be used. For the simplicity of the Finite Element Method (FEM) it is good to choose a geometry with known boundary conditions. This can be performed by insulating certain boundaries or controlling the temperature at certain boundaries. A combination of this has been used in the chosen geometry. Eighteen thermocouples are placed in the SMC material in order to verify the temperatures in the physical test setup compared with the simulations. The thermocouples are positioned in such a way that there are always two sensors that should have the same position and therefore the same temperature. In order to get a high accuracy of the collected data these two measured values are therefore used to calculate an average. The collected data can then be used to compare with simulated values. There are also two thermocouples positioned on top of the litz wire in order to measure the coil temperature. Earlier, motors have been tested, simulated and evaluated with these materials, but due to the complexity of those machines a simpler case is chosen. Electromagnetic fields are used in both motors, induction 
heaters [14]-[16] and filter inductors, as well as the same type of materials. An induction heater cell is therefore chosen to perform the tests on.

The first thing to do is to make sure that the losses in the litz wire are correct in the simulation. This is done by measuring the electric properties of a coil with the same geometry as the one used in the other experiments, but without any SMC material or load. The measurement was performed by measuring the resistance on the coil with an LCR meter at the intended frequencies. The difference in powers between the simulation and the measurements are then used in order to compensate any deviation in the simulation for the coil with SMC material. The length of the connection ends of the coil is also compensated for by calculating the percentage of the connection ends in relation to the coil and then subtracted from the resistance measured.

In order to obtain the uncertain narrowed parameters, several different tests will be performed. In setup 1 only the inductor and no work piece will be used. This will be tested using DC current in order to validate the thermal conductivity model and the resistive losses in the litz wire on DC. AC tests at several frequencies and powers will also be performed in this setup in order to determine the losses in the SMC. In setup 2 a work piece will be used on AC tests.

The thermal conductivity of the litz wire will be predicted by using the Hashin and Shtrikman approximation, Equation (1).

$$
k_{e}=k_{p} \frac{\left(1+\vartheta_{c u}\right) \times k_{c u}+\left(1-\vartheta_{p}\right) \times k_{p}}{\left(1-\vartheta_{c u}\right) \times k_{c u}+\left(1+\vartheta_{p}\right) \times k_{p}}
$$

where $k$ and $\vartheta$ are the thermal conductivity and volumetric fraction with the subscripts $e, p$ and $c u$ representing the effective, potting and copper, respectively. The usage of Equation (1) is not entirely correct due to the twisted copper wires in the litz wire, but is considered to be close enough.

The losses in the SMC will be predicted by using the Steinmetz Equation (2).

$$
P=k f^{\alpha} B^{\beta}
$$

where $P$ is the power loss $\left(\mathrm{W} \cdot \mathrm{m}^{-3}\right), k, \alpha$ and $\beta$ the fitting coefficients, $f$ the frequency $(\mathrm{Hz})$, and $B$ the peak magnetic flux density $(\mathrm{T})$.

In the experiments a fixed temperature will be set to the cooling liquid, as well as a set current and frequency, see Table 1 . The equipment used is voltage controlled, which

\begin{tabular}{|c|c|c|c|c|c|c|c|}
\hline \multirow[b]{2}{*}{ Frequency $[\mathrm{kHz}]$} & \multicolumn{4}{|c|}{ Setup 1} & \multicolumn{3}{|c|}{ Setup 2} \\
\hline & DC & 15 & 20 & 25 & 15 & 20 & 25 \\
\hline \multicolumn{8}{|c|}{ Current RMS [A] } \\
\hline Test 1 & 40 & 29.5 & 25.4 & 22.7 & 35.2 & 29.7 & 25.5 \\
\hline Test 2 & 45 & 35.7 & 29.8 & 30.7 & 39.4 & 32 & 29.9 \\
\hline Test 3 & 50 & 41.3 & 32.6 & 38.1 & 44.4 & 38.1 & 34.6 \\
\hline Test 4 & 55 & 44.7 & 36.7 & 42.5 & 48.6 & 42.8 & 41 \\
\hline Test 5 & 60 & 48 & 42 & 46.9 & 51.5 & 48.9 & 44.9 \\
\hline
\end{tabular}

Table 1. Experimental parameters. 
means that it is difficult to set a specified current. The currents are specified so that the temperature on top of the litz wire will be relatively evenly distributed from $120^{\circ} \mathrm{C}$ to $40^{\circ} \mathrm{C}$. The currents are therefore different for different measurements. The setup will run until a steady state is reached and the temperatures will be measured. This will then be performed for several currents and frequencies in order to compare with simulated results.

\section{Simulation Model}

In order to simulate the test setup a FEM program that can handle nonlinear multiphysics problems is needed. It is also very important to be able to correctly simulate the high frequency losses in the litz wire. Finite Element Method Magnetics 4.2 [FEMM] is one simulation software that can handle this, and is therefore chosen [17]-[23]. The FEMM software is controlled from MATLAB by commands given in the FEMM documentation in order to combine the heat and magnetic simulations. The losses generated in the magnetic simulation are transferred to the heat simulation in order to generate heat losses required to determine the temperature pattern. The losses generated in the litz wire is calculated in the FEMM software and described by Meeker in [20]. Since the resistivity of the litz wire is temperature dependent [24], the program need to iterate between the two simulation models. Convergence is assumed when the total loss increase for a new iteration is below $0.1 \mathrm{~W}$. In order to get the correct iron losses in the SMC the Steinmetz coefficients are used. By extracting the flux density of individual positions in the SMC material from the magnetic simulation, the iron loss density can be calculated. The result is then incorporated in the heat simulation model. The iron losses are also temperature dependent, but in such small degrees that it is neglected in the simulations.

It is not possible to transfer losses on an element level from one simulation to the other in FEMM 4.2. It has to be done by transferring a value to a whole area. Calculating the loss for the whole SMC and then transfer the value to the entire area would not be correct because the losses is generated in different parts of the SMC depending on the magnetic flux concentration. The geometry of the SMC is instead divided into smaller regions in the thermal simulation where the losses are transferred to. Thus, a simple adaptive algorithm that uses the loss gradient to decide the size of each area created is used to transfer the losses from the magnetic simulation to the thermal simulation, Figure 2. Since the flux density of any given cooridinate can be obtained from the program based on interpolated values, the loss $W_{i}$ of each rectangular region limited by the corners $\left[\begin{array}{ll}x_{i} & y_{j}\end{array}\right]$ and $\left[\begin{array}{ll}x_{i+1} & y_{j+1}\end{array}\right]$ is calculated according to Equations (3) and (4).

$$
\begin{aligned}
W_{i, j} & =\sum_{x_{i}}^{x_{i+1}} \sum_{y_{j}}^{y_{j+1}} W(B(x, y)) / A_{i} \\
A_{i} & =\left(x_{i+1}-x_{i}\right) \cdot\left(y_{j+1}-y_{j}\right)
\end{aligned}
$$

Different loss gradient values have been tested in order to create a correct and 
efficient model. The mesh size has also been optimized in a similar way. During testing it was found that the model is not sensitive to the change in mesh size and the choice of loss gradient values. The number of element used for the electromagnetic and thermal simulation respectively are about 618,000 and 320,000 . In the thermal simulation, the SMC material was divided into approximately 500 regions of different size according to Figure 2.

The temperature in the litz wire is going to be different in different turns. The 22 boxes that makes up the litz wire is therefore considered to have different temperatures. The losses is therefore integrated over each individual box and transferred to the corresponding turn in the thermal simulation. The temperature is then transferred back for each box individually to the magnetic simulation for each iteration.

Two different models are used in order to evaluate every different important parameter. The first setup is without any work piece, Figure 1. It is cooled at the bottom by a constant temperature and insulated at the sides and top. The simulation is performed using both $\mathrm{AC}$ and $\mathrm{DC}$ currents in order to investigate the different properties. Firstly, DC is used to investigate the thermal conductivity of the litz wire and the rest of the model, without any losses in the SMC material and only the resistive losses in the wire. Secondly, AC is used to investigate the iron losses created in the SMC. Also AC losses in the litz wire due to skin and proximity effects are considered in this case.

The second setup has an aluminum work piece and uses high frequency AC currents, Figure 2.

Both models are run at different powers and the AC simulations run at different frequencies, see Table 1 and Table 2.

The Steinmetz coefficients used to calculate the loss in the SMC is; $k=67.0 ; \alpha=1.33$; $\beta=1.93$.
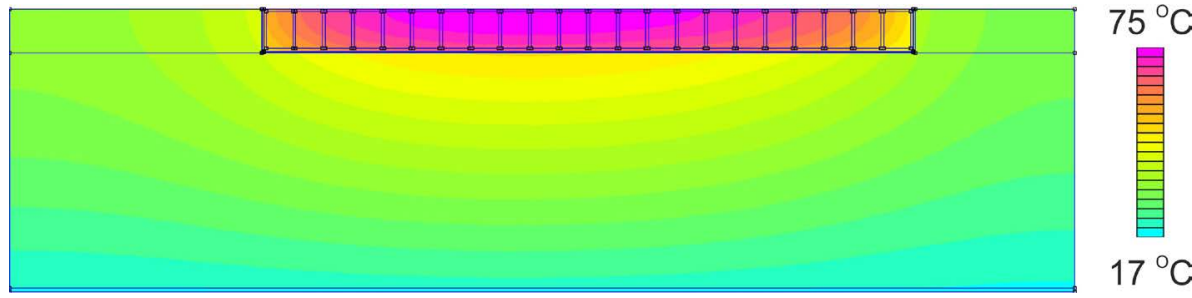

Figure 1. Heat simulation of the inductor without work piece on DC.
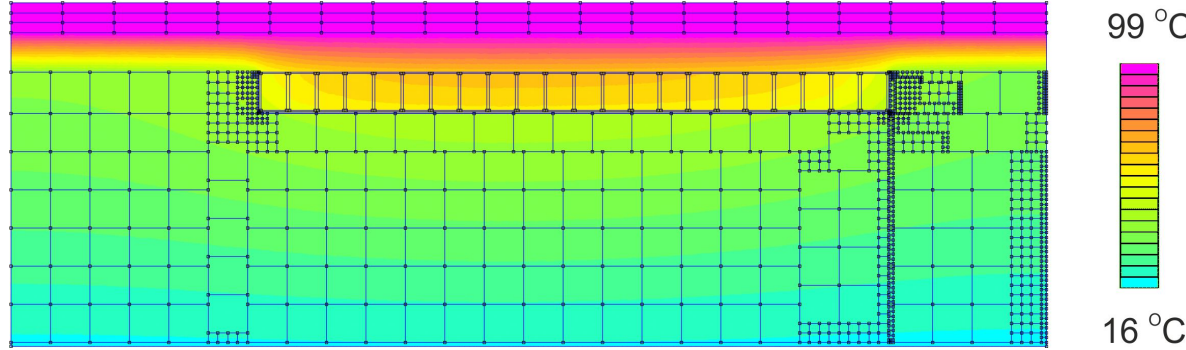

$16{ }^{\circ} \mathrm{C}$

Figure 2. Heat simulation of the inductor with work piece at $15 \mathrm{kHz}$. 
Table 2. Material properties used in simulations.

\begin{tabular}{cccccccc}
\hline Material/Property & Air & Litz & SMC & Al 5052 & Superwool & Epoxy & Kapton \\
\hline Relative permeability [-] & 1 & 1 & 16 & 1 & 1 & 1 & 1 \\
$\quad \begin{array}{c}\text { Electrical } \\
\text { conductivity @ } 20^{\circ} \mathrm{C}[\mathrm{MS} / \mathrm{m}]\end{array}$ & 0 & 54 & 0 & 20 & 0 & 0 & 0 \\
$\begin{array}{c}\text { Electrical temperature } \\
\text { coefficient }\left[\mathrm{K}^{-1}\right]\end{array}$ & - & 0.00394 & - & - & - & - & - \\
$\quad \begin{array}{c}\text { Volumetric heat } \\
\text { capacity }\left[\mathrm{MJ} /\left(\mathrm{m}^{3} \mathrm{~K}\right)\right]\end{array}$ & 0.0012 & 2.6 & 2.5 & 2.35 & 0.01 & 1.7 & 1.7 \\
$\quad \begin{array}{c}\text { Thermal } \\
\text { conductivity }[\mathrm{W} /(\mathrm{m} \mathrm{K})]\end{array}$ & 0.025 & 0.85 & 2.2 & 138 & 0.06 & 0.2 & 0.2 \\
\hline
\end{tabular}

In the simulations it has been observed that only a very low percentage of the heat is dissipated through the insulation on the side and the top. Therefore, it is considered to be a good approximation to set the boundary condition to perfectly insulated on both the top and the sides. The work piece in the second setup is an aluminum pot with boiling water in. The boundary condition is therefore approximated to a constant temperature of $100^{\circ} \mathrm{C}$ on top of the work piece.

How sensitive are the results if a parameter is incorrect? Data that are supplied from a manufacturer might not be exactly correct, and data that are measured or calculated on a sample might also deviate from the correct value. In order to know how variations of different material parameters will affect the results, simulations where one parameter at a time is changed plus and minus $10 \%$ will be compared and discussed.

\section{Experiments}

The tests are performed in three steps in order to get all the data verified. In the first step we use only the inductor with the cooling plate on the bottom and $90 \mathrm{~mm}$ insulation on the sides and on top, Figure 3. At this point all measurements are performed with DC. By using DC no iron losses will occur in the SMC material but only resistive losses in the litz wire.

The collected data can then be used to compare with simulated values from FEMM. The aluminium cooling block is glued to the bottom with epoxy, and the DC test are therefore used to calibrate the thickness of the epoxy in the FEMM simulation.

The second step is to run the same setup as before with AC instead of DC. By running it with $\mathrm{AC}$, the iron losses in the SMC material can be calculated.

The third step is to run the same setup with AC and a load, in this case an aluminum pot filled with boiling water on top of the heater, see Figure 4.

The litz wire used in the test setup consists of 192 individually insulated wires with a copper diameter of $0.2 \mathrm{~mm}$. It has insulation around the entire wire consisting of textile yarn, which gives it a total outer dimension of $3 \times 4 \mathrm{~mm}$. The coil consists of 22 turns of litz wire in one layer. After winding, the wire is fully impregnated with epoxy in order to increase the thermal conductivity, as well as ensuring it is mechanically stable.

In this experimental setup there are thermocouples of $\mathrm{K}$ type with accuracy of $\pm 1.5^{\circ} \mathrm{C}$. 


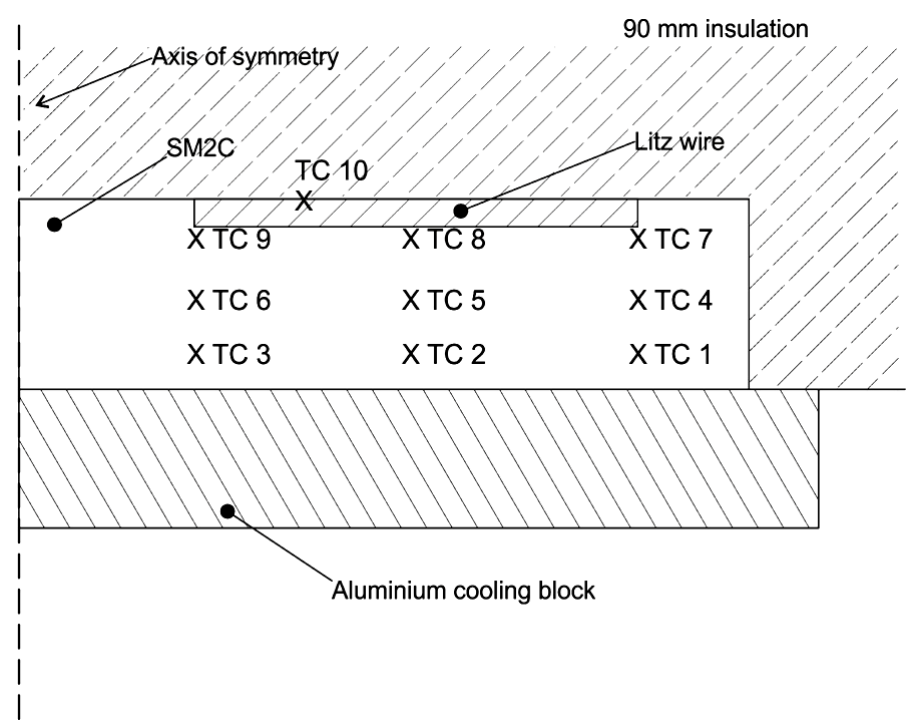

Figure 3. Thermocouple placement on a schematic test setup 1 without load.

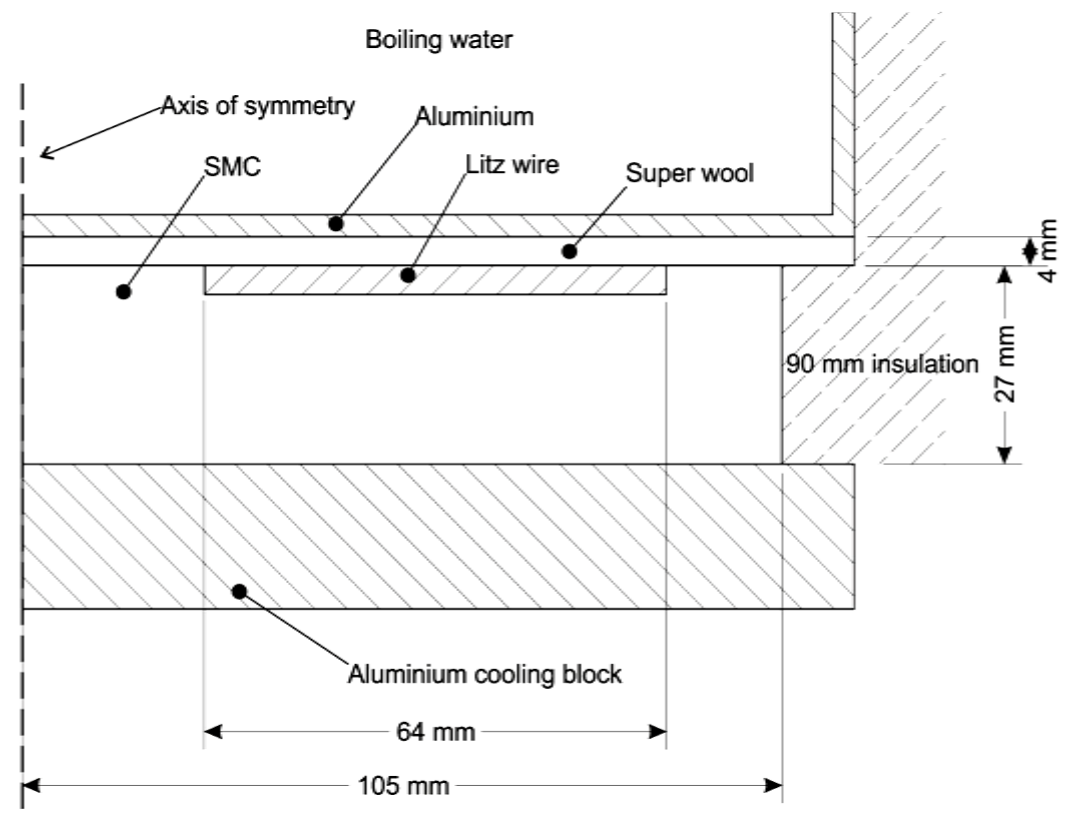

Figure 4. Schematic of test setup 2 with aluminum load.

In order to minimize the temperature measurement uncertainty, all the thermocouples are tested and calibrated at room temperature before each test.

The positioning of the thermocouples is very important. It is possible to mold the thermocouples directly into the composite; however, it is difficult to verify where the sensors are positioned after mounting. Since it is critical to position them in a predefined position, the mounting is made after molding by drilling holes in the now molded composite and mounting the sensors in the holes. The drill has a 118 degree angle on the tip, which makes it easy to place the thermocouple in the center of the hole. The holes are then molded with SMC. 
The holes are drilled from beneath, impossible to place the thermocouples wrongly in the radial direction, as long as they are placed in the bottom of the hole. However, it is possible that the thermocouples will be axially misplaced. The incorrectly placed thermocouple will always have a lower temperature than the correctly placed. If two thermocouples that should have the same temperature deviate too much from each other the thermocouple with the highest temperature is assumed to be the correct one.

The positioning of the two thermocouples on the litz wire is not that critical. In simulations it can be seen that the temperature only has relatively small variations over the wire where the thermocouples are positioned.

\section{Results and Discussion}

When comparing the simulated results with the experimental results for the DC case, a good agreement can be seen. The difference between the simulated results and the experimental results can be seen in Table 3, and the results for the $50 \mathrm{~A}$ test are illustrated in Figure 5.

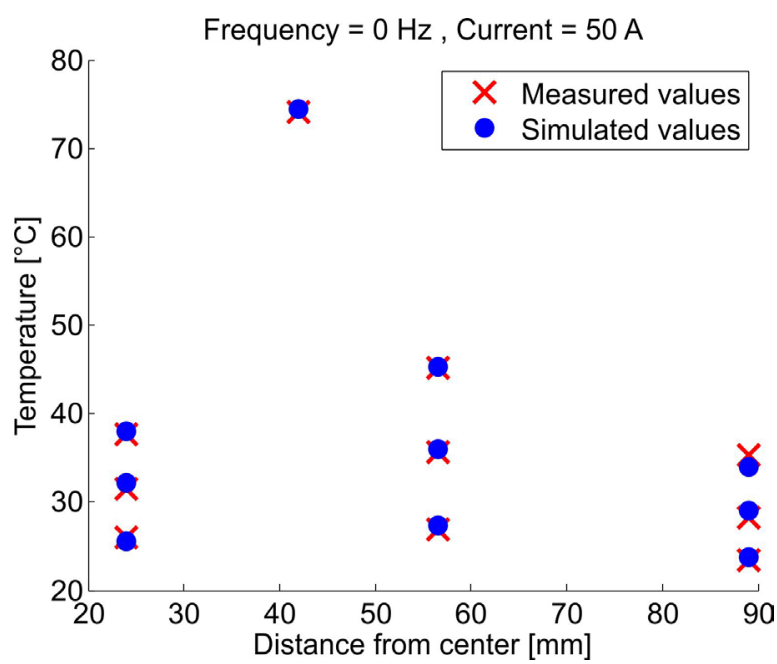

Figure 5. Comparison between simulated and measured temperatures at 50 A DC.

Table 3. Temperature deviation in degrees $\mathrm{K}$ between simulated and measured values. The numbering of the thermocouples correlate with the numbering in Figure 3.

\begin{tabular}{cccccc}
\hline & $40 \mathrm{~A}$ & $45 \mathrm{~A}$ & $50 \mathrm{~A}$ & $55 \mathrm{~A}$ & $60 \mathrm{~A}$ \\
\hline TC 1 & 1.99 & 1.73 & 1.41 & 0.55 & -0.09 \\
TC 2 & 2.16 & 1.81 & 1.71 & 0.99 & 0.11 \\
TC 3 & -0.14 & -0.90 & -1.50 & -2.73 & -4.16 \\
TC 4 & 2.40 & 2.59 & 2.82 & 2.46 & 1.95 \\
TC 5 & 1.31 & 1.12 & 1.13 & 0.10 & -1.66 \\
TC 6 & 2.26 & 2.18 & 2.14 & 1.51 & 0.63 \\
TC 7 & -3.04 & -3.45 & -4.00 & 4.62 & -5.53 \\
TC 8 & 0.65 & 0.84 & 0.50 & -0.17 & -1.06 \\
TC 9 & 1.46 & 1.31 & 1.12 & 0.18 & -1.12 \\
TC 10 & 0.92 & -0.35 & 0.48 & -1.72 & -6.55 \\
\hline
\end{tabular}


When looking at sensor 10, the first/lowest four currents show good agreement between the simulations and the experiments; however, the last/highest current, $60 \mathrm{~A}$, shows a lower value in the simulation compared to the experimental reading. An explanation could be that the thermal conductivity is measured at room temperature, and the elevated temperature could change the properties for the materials. However, there is good agreement between the simulated and the experimental results for the other nine sensors in all measurements. Therefore, the simulation model is seen as a good representation of the experimental setup at temperatures up to $70^{\circ} \mathrm{C}$ with $\mathrm{DC}$ current excitation.

Adding complexity to the tests and simulations by using AC currents and an optional induction load, that is water-cooled aluminum sheet. Figure 6(a) shows a comparison

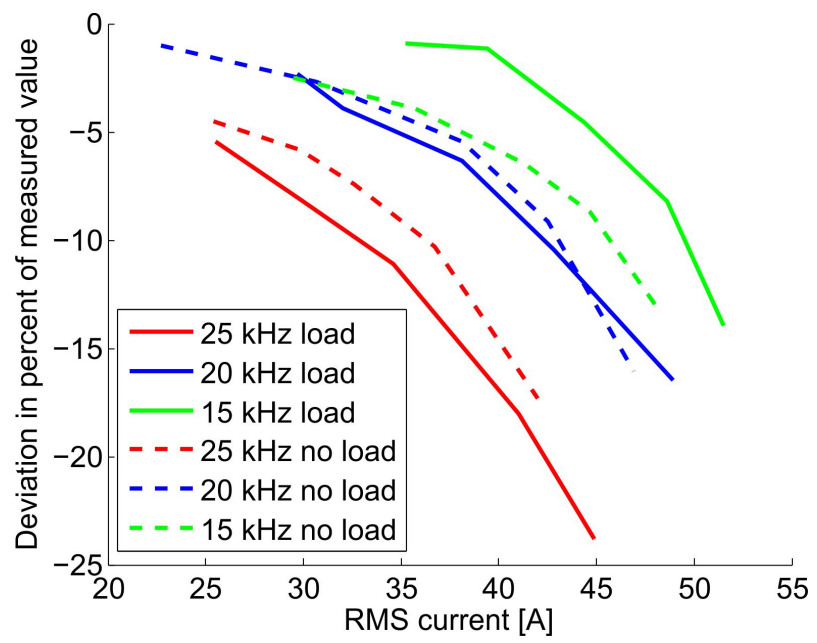

(a)

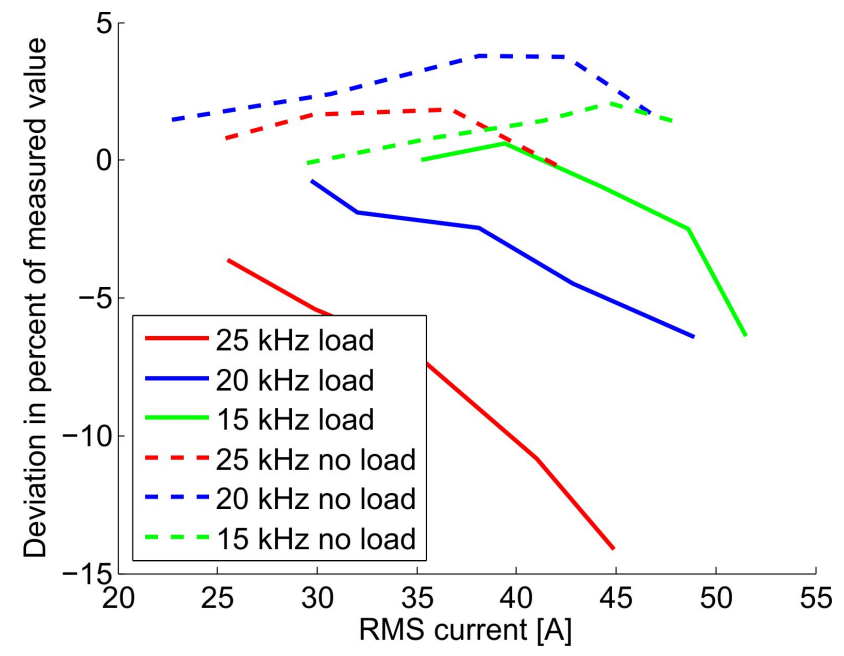

(b)

Figure 6. Comparison of deviation between simulation and measured values, with and without aluminum load. The measuring point chosen is the one on top of the litz wire, Sensor 10. (a) Before calibration; (b) After calibration. 
between the relative error of temperature sensor 10 and the simulated temperature for that location when using AC currents in the coil, with and without induction loading.

It is clear from Figure 6(a) that the simulated temperature of the litz wire is much lower than the measured one, and the deviation increases with increased current. The reason for this is unknown. The simulation should take into account the skin effect and the proximity losses. The capacitive/dielectric losses have been measured and calculated, but are less than one percent of the total losses and can be neglected.

A typical temperature point comparison can be seen in Figure 7(a), where it is obvious that the temperatures in the SMC behaves according to the model, while the temperature of the litz wire shows a considerable error. In order to work around this problem a simulation of only the coil without any load or SMC material is performed. The deviation between simulation and measurement is used to calibrate the losses in

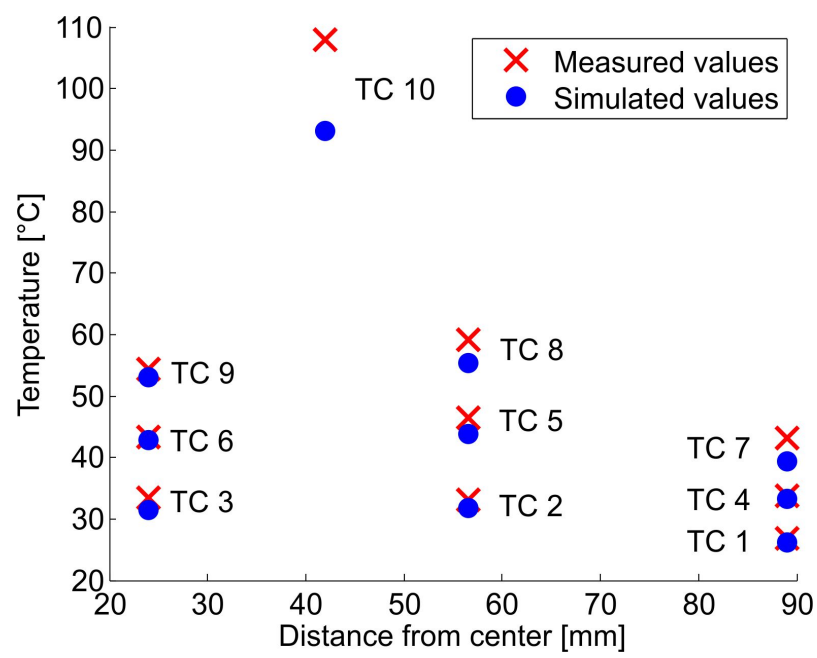

(a)

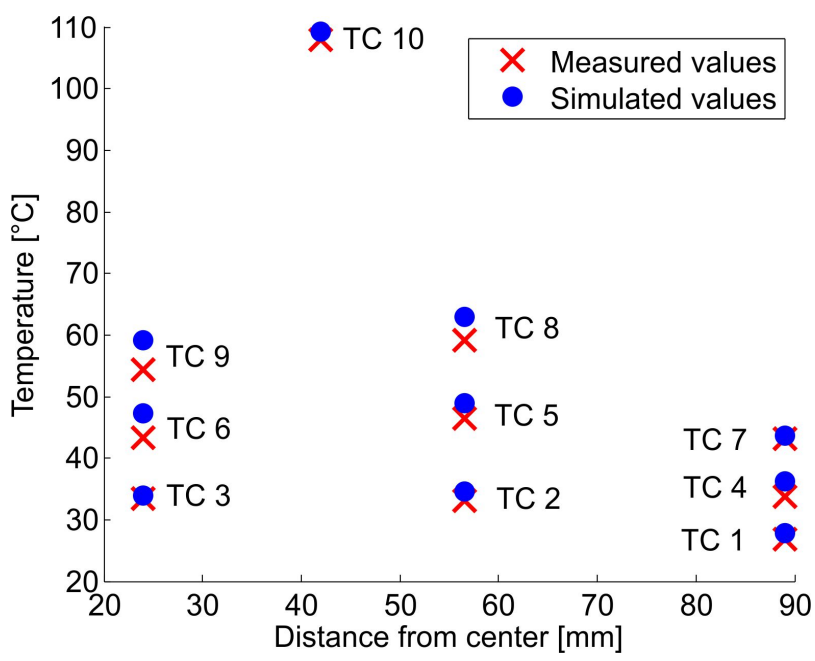

(b)

Figure 7. Comparison of measured values with simulated values at $15 \mathrm{kHz}$ and $48 \mathrm{~A}$ without induction load. (a) Uncompensated simulated values; (b) Compensated simulated values. 
the wire by increasing its power loss. The results after calibration can be seen in Figure 6(b).

It is clear that the calibration works well for the non-loaded case, while the errors of the loaded case are reduced by approximately $50 \%$. This is because the proximity effect will affect the losses. A representative figure for the non-loaded case can be seen in Figure 7(b).

When looking at Figure 7(a) and Figure 7(b), it is clear that the losses in the litz wire to a high degree affect the temperature in the SMC material since the calibration just added loss to the wire. It is then possible to see that with the correct, calibrated loss in the litz wire, the temperature in the SMC material coincides with simulation and measurement. This means that the simulation of the losses in the SMC material is essentially correct.

The losses in the SMC are between $22 \%$ - 32\% of the total loss in the induction heater when no induction load is used. The losses in the SMC have decreased to less than $1 \%$ with load. This is mainly because of the increased reluctance of the magnetic circuit with the aluminum sheet induction loading. The efficiency of the induction heater with aluminum load is above $72 \%$ for all measured cases, see Table 4 .

A comparison of the temperature deviation in the litz wire, sensor 10, is shown in Figure 6(a). It can be seen that the temperature deviation is dependent of the frequency and with or without induction loading. The increase of deviation with the increase of the current is most likely due to the thermal model deviation seen in the DC case at elevated temperatures, while the deviation due to the frequency depends on the conservative power loss model of the litz wire.

A sensitivity analysis has been performed for seven key parameters in the simulation. Figure 8 shows how much the temperature on top of the litz wire changes if one parameter at a time is changed plus/minus $10 \%$. A similar behavior can be observed for all measuring points in the simulation.

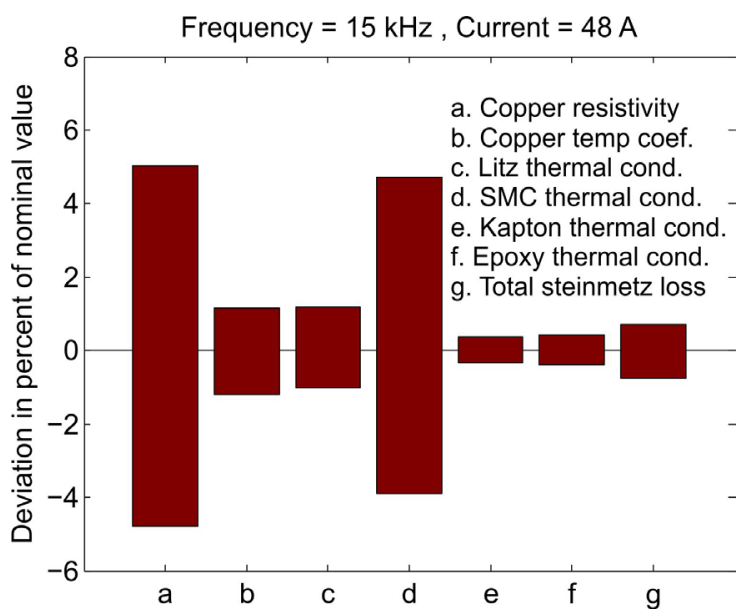

Figure 8. Sensitivity analysis of seven key parameters in the simulation model, performed without load and compensation of losses. The temperature from the simulation used is the one on top of the litz wire, sensor 10. 
Table 4. Power losses in watt in the different parts of the induction heater.

\begin{tabular}{ccccc}
\hline- & Litz wire & cooling block & Aluminum load & SMC \\
\hline DC, 50 A & 70.9 & - & - & - \\
AC, $15 \mathrm{kHz}, 48$ A, without load & 68.7 & 10.4 & - & 27.5 \\
AC, $15 \mathrm{kHz}, 51.5$ A, with load & 90.1 & 0.06 & 254.5 & 1.97 \\
\hline
\end{tabular}

It is clear that the litz wire's copper resistivity and the thermal conductivity of the SMC are the most influential properties, thus having the largest impact on the simulation errors. The losses in the SMC only have a minor effect on the temperatures in the inductor. This of course depends on which SMC material is used.

In practice, it is hard to change the copper resistivity, but it is possible to increase the cross sectional copper area, and thereby lower the current density. This can be done by either increasing the space for the litz wire or increasing the packing factor.

The thermal conductivity of the SMC is highly dependent on the SMC type. In this case, some kind of thermal conductive filler in the epoxy or by increasing the packing density of the powder should increase the bulk thermal conductivity. Thus, it is important that the ferromagnetic packing density of the powder is not decreased in the search for a higher thermal conductivity.

\section{Conclusions}

The simulated temperatures show good agreement with the measured values, which means that it is possible to model the whole system with coupled simulations. Another conclusion from these results is that the input parameters are accurate and robust, in other words the methods and procedures to obtain critical material properties for SMC and litz wires are equally accurate and robust.

A key parameter for induction heaters and filter inductors is the service temperature. The heat from losses will be generated inside the inductor itself, whereas it is critical to effectively transport the heat away from the inductor core. A high thermal conductivity is therefore important in the litz wire and in the SMC material. One conclusion from the studied setup in this paper is that the thermal conductivity of the SMC is more important than the thermal conductivity of the litz wire's structure. Generally, this condition depends on the materials used in each case, as well as the current frequency and the geometry of different parts. However, it shows the importance of the thermal properties of the material used, not only the electrical and magnetic properties.

The results of the study also indicate which material properties would be the most beneficial to improve. In the studied case it is obvious that the material development of the SMC should be focused on thermal conductivity. Improvement of the litz wire's structure should be focused in lower losses, which could be obtained by increasing the cross section, or rather by introducing thinner strands.

\section{Acknowledgements}

The present work was carried out with the generous support of the EU-program 
Interreg VA Nord, (project NorFaST-HT Nordic Community for Fast Steel Heat Treatments), within the Sustainable Production Initiative (SPI Vinnova) and within the Center for ElectroMagnetic Energy Conversion (CEMEC).

\section{References}

[1] Monier-Vinard, E., Bissuel, V., Dia, C.T., Daniel, O. and Laraqi, N. (2013) Investigation of Delphi Compact Thermal Model Style for Modeling Surface-Mounted Soft Magnetic Composite Inductor. IEEE 19th International Workshop on Thermal Investigations of ICs and Systems (THERMINIC), Berlin, 25-27 September 2013, 315-318. http://dx.doi.org/10.1109/THERMINIC.2013.6675210

[2] Wrobel, R., McNeill, N. and Mellor, P.H. (2010) Performance Analysis and Thermal Modeling of a High-Energy-Density Prebiased Inductor. IEEE Transactions on Industrial Electronics, 57, 201-208.

[3] Guo, Y., Zhu, J. and Zhong, J. (2006) Measurement and Modelling of Magnetic Properties of Soft Magnetic Composite Material under 2d Vector Magnetisations. Journal of Magnetism and Magnetic Materials, 302, 14-19.

[4] Bozorth, R.M. (1993) Ferromagnetism. Wiley-VCH, Hoboken, 992.

[5] ISO, S. (2008) Plastics-Determination of Thermal Conductivity and Thermal Disutility: Part 2.

[6] Zhang, D. and Foo, C. (1999) A Simple Method to Estimate the Magnetic Field Distribution due to Current-Carrying Winding in Toroidal Core and Its Influence on the Measurement of Complex Permeability and Core Losses. Journal of Magnetism and Magnetic Materials, 191, 189-198.

[7] Gustavsson, M., Nagai, H. and Okutani, T. (2007) Characterization of Anisotropic and Irregularly-Shaped Materials by High-Sensitive Thermal Conductivity Measurements. In: Ahn, B.T., Jeon, H., Hur, B.Y., Kim, K. and Park, J.W., Solid State Phenomena, Vol. 124, Trans Tech Publications, Pfaffikon, 1641-1644.

[8] Claassen, J. (2006) A Novel Technique for Measurement of Core Loss in Low Permeability Materials. Journal of Magnetism and Magnetic Materials, 301, 541-545.

[9] Henrich, F., Rahn, H. and Odenbach, S. (2014) Investigation of Heat Distribution during Magnetic Heating Treatment Using a Polyurethane-Ferrouid Phantom-Model. Journal of Magnetism and Magnetic Materials, 351, 1-7. http://dx.doi.org/10.1016/j.jmmm.2013.09.046

[10] Shazly, J. and Adly, A.A. (2012) Extensions to the Finite Element Technique for the Magneto-Thermal Analysis of Aged Oil Cooled-Insulated Power Transformers. Journal of Electromagnetic Analysis and Applications, 4, 167. http://dx.doi.org/10.4236/jemaa.2012.44022

[11] Babaie, H. and Farahani, H.F. (2010) Analysis of Thermal Behavior of High Frequency Transformers Using Finite Element Method. Journal of Electromagnetic Analysis and Applications, 2, 627-632.

[12] Svensson, L., Frogner, K., Jeppsson, P., Cedell, T. and Andersson, M. (2012) Soft Magnetic Moldable Composites: Properties and Applications. Journal of Magnetism and Magnetic Materials, 324, 2717-2722. http://dx.doi.org/10.1016/j.jmmm.2012.03.049

[13] Siesing, L., Reinap, A. and Andersson, M. (2014) Thermal Properties on High _ll Factor Electrical Windings. IEEE International Conference on Electrical Machines (ICEM), Berlin, 2-5 September 2014, 2218-2223.

[14] Lucia, O., Maussion, P., Dede, E.J. and Burdio, J.M. (2014) Induction Heating Technology and Its Applications: Past Developments, Current Technology, and Future Challenges. 
IEEE Transactions on Industrial Electronics, 61, 2509-2520.

[15] Rudnev, V., Loveless, D., Cook, R.L. and Black, M. (2002) Handbook of Induction Heating. CRC Press, New York.

[16] Frogner, K., Svensson, L., Andersson, M., Cedell, T. and Stahl, J.-E. (2011) A New Concept of Coil Design for Industrial Induction Heating. SPS11, 4th Swedish Production Symposium, Lund, 3-5 May 2011, 501-509.

[17] Shibaji, B., Siddhartha Shankar, P., Partha, B. and Sabyasachi, M. (2013) Pulsed Magnetic Field Measurement outside Finite Length Solenoid: Experimental Results \& Mathematical Verification. Journal of Electromagnetic Analysis and Applications, 5, 371-378.

[18] Frogner, K., Cedell, T. and Andersson, M. (2014) Decoupling of Currents in Travelling Wave Induction Heating. Journal of Electromagnetic Analysis and Applications, 6, 193-202.

[19] Muhlethaler, J., Kolar, J.W. and Ecklebe, A. (2011) Loss Modeling of Inductive Components Employed in Power Electronic Systems. IEEE 8 th International Conference on Power Electronics and ECCE Asia (ICPE \& ECCE), 30 May-3 June 2011, 945-952. http://dx.doi.org/10.1109/icpe.2011.5944652

[20] Meeker, D.C. (2012) An Improved Continuum Skin and Proximity Effect Model for Hexagonally Packed Wires. Journal of Computational and Applied Mathematics, 236, 4635 4644.

[21] Roy, M. and Sengupta, M. (2013) Electromagnetic Investigations and Power Converter Efficiency Studies on a Laboratory Made Induction Heating Prototype. Journal of The Institution of Engineers (India): Series B, 94, 153-160.

[22] Makni, Z. and Demersseman, R. (2014) A Coupled Analytical-Numerical Approach for Optimal Sizing of Power Inductors. COMPEL: The International Journal for Computation and Mathematics in Electrical and Electronic Engineering, 33, 1653-1664. http://dx.doi.org/10.1108/COMPEL-12-2013-0411

[23] Pusz, A. and Trojnacki, Z. (2012) The Modelling of Thermal Conductivity Measurements Using FEMM Application. Archives of Materials Science and Engineering, 53, 53-60.

[24] Dellinger, J. (1910) The Temperature Coefficient of Resistance of Copper. Journal of the Franklin Institute, 170, 213-216.

\section{Scientific Research Publishing}

\section{Submit or recommend next manuscript to SCIRP and we will provide best service for you:}

Accepting pre-submission inquiries through Email, Facebook, LinkedIn, Twitter, etc. A wide selection of journals (inclusive of 9 subjects, more than 200 journals)

Providing 24-hour high-quality service

User-friendly online submission system

Fair and swift peer-review system

Efficient typesetting and proofreading procedure

Display of the result of downloads and visits, as well as the number of cited articles

Maximum dissemination of your research work

Submit your manuscript at: http://papersubmission.scirp.org/

Or contact jemaa@scirp.org 